\title{
Correction to: Inactivation of ICAM1 inhibits metastasis and improves the prognosis of Ewing's sarcoma
}

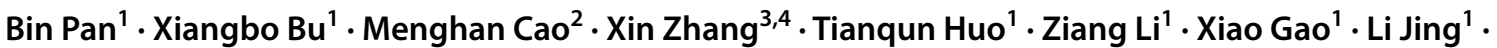 \\ Xuanxiang Luo ${ }^{1} \cdot$ Hu Feng $^{1} \cdot$ Feng Yuan $^{1} \cdot$ Kaijin Guo $^{1}$ (])
}

Published online: 6 January 2022

๑) Springer-Verlag GmbH Germany, part of Springer Nature 2021

\section{Correction to: Journal of Cancer Research and Clinical Oncology (2021) 147:393-401 \\ https://doi.org/10.1007/s00432-020-03431-3}

In the original article, the Fig. 5 is published incorrectly. The correct figure is given below.
The original article has been corrected.

Publisher's Note Springer Nature remains neutral with regard to jurisdictional claims in published maps and institutional affiliations.

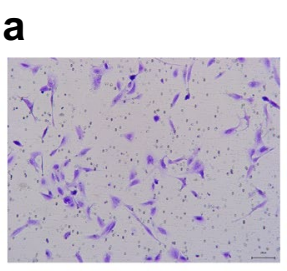

si-ICAM1

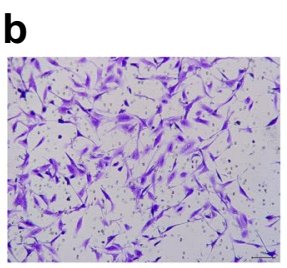

Lenti-ICAM1

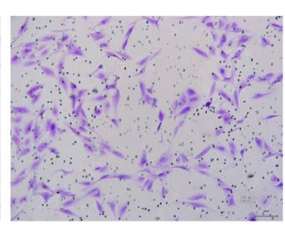

si-Control

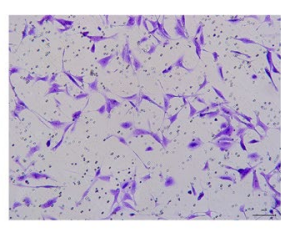

Lenti-Control

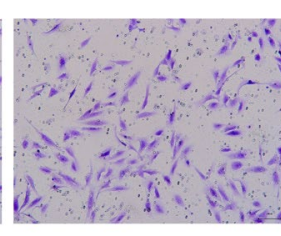

Control
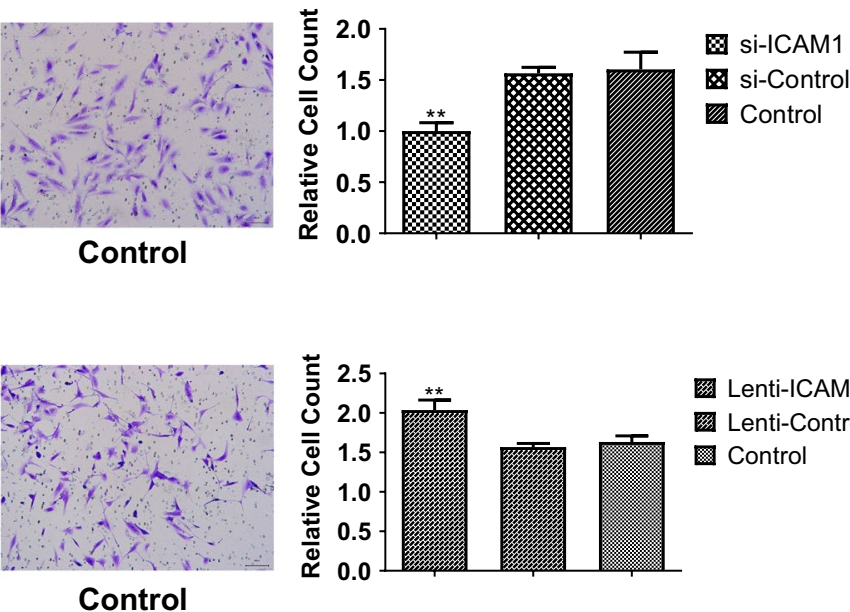

国 Lenti-ICAM1

四 Lenti-Control

Control

The original article can be found online at https://doi.org/10.1007/ s00432-020-03431-3.

\section{Hu Feng}

fenghuxz@163.com

$\triangle$ Feng Yuan

yuanfengxz@yeah.net

$\triangle$ Kaijin Guo

guokaijinxz@yeah.net

1 Department of Orthopedics, The Affiliated Hospital of Xuzhou Medical University, Xuzhou 221000, China

2 Department of Oncology, The Affiliated Hospital of Xuzhou Medical University, Xuzhou 221000, China
3 Department of Dermatology, Xuzhou Children's Hospital, Xuzhou Medical University, Xuzhou 221000, China

4 Department of Dermatology, The Affiliated Hospital of Xuzhou Medical University, Xuzhou 221000, China 